Abstract

\title{
Structural Health Monitoring Using Carbon Nanotube Yarns: Sensing Concept and Applications in Composites ${ }^{+}$
}

Jandro L. Abot

Department of Mechanical Engineering, The Catholic University of America, Washington, DC 20064, USA; abot@cua.edu

+ Presented at the 5th International Symposium on Sensor Science (I3S 2017), Barcelona, Spain, 27-29 September 2017.

Published: 4 December 2017

Non-destructive evaluation and structural health monitoring techniques can provide frequent or immediate feedback of the condition of a structure including potential damage. However, these techniques cannot detect initiating damage in composite materials with high compaction or multifaceted construction. More critically, they are unable to achieve damage detection without altering the microstructure of the composite material. An alternative method of strain monitoring and damage detection that may offer the advantages of structural health monitoring without their drawbacks consists of using piezoresistive-based carbon nanotube (CNT) yarns integrated in polymers and composite materials. The concept is that the CNT yarns form a continuous sensor circuit and their inherent piezoresistive sensitivity detects strain within the host material through resistance measurements without adding much weight or altering the integrity of the host material. This presentation includes a summary of the piezoresistive response of CNT yarns and the concept and latest experimental results on damage detection in laminated polymeric composite materials and distributed and localized strain measurement. Experimental results also show the ability of a combination of different yarn sensors to detect the exact location and extent of delamination in real time. CNT yarn sensors may provide an adaptive, practical, and sensitive structural health monitoring technique.

(C) 2017 by the authors. Licensee MDPI, Basel, Switzerland. This article is an open access article distributed under the terms and conditions of the Creative Commons Attribution (CC BY) license (http://creativecommons.org/licenses/by/4.0/). 